Dhaka Univ. J. Biol. Sci. 25(2): 161-167, 2016 (July)

\title{
ADAPTATION OF STIRLING CHILDREN'S WELL-BEING SCALE (SCWBS) IN BANGLADESH CONTEXT
}

\author{
Mahjabeen Haque* AND Mostak Ahamed ImRan \\ Department of Educational and Counselling Psychology, University of Dhaka, \\ Dhaka-1000, Bangladesh
}

Key words: Adaptation, Children's well-being, Scale adaptation

\begin{abstract}
The purpose of this study was to adapt Stirling Children's Well-Being Scale (SCWBS) for measuring well-being of children of Bangladesh. Data were collected from 238 students, ages between 10 to 16 years of three different schools of Dhaka city. Psychometric properties were measured through Cronbach's alpha (0.746), split-half (0.782), test-retest (0.791), which were satisfactory, respectively at $0.05,0.05$, and 0.01 level of significance. The demonstration of construct validity with Beck Self-Concept Inventory for Youth (BSCI-Y) and Beck Anxiety Inventory for Youth (BAI-Y) (two subtests of Adapted Bangla Beck Youth Inventories of Emotional and Social Impairment Scale) were 0.668 and -0.350 , respectively, and item-total correlation ( 0.258 to 0.451) was also satisfactory. Thus psychometric results support that Bangla SCWBS is suitable for use in Bangladesh context. Mental health professionals can use Bangla SCWBS as a useful tool to assess changes in children's wellbeing from a positive psychological perspective.
\end{abstract}

\section{Introduction}

Well-being is a term for the state of an individual or group, for example their emotional, psychological, social, spiritual or medical state, etc. There is no exact definition for the term 'well-being' in literature(1). Well-being is a socially constructed concept; that is, each society, group, culture, institution and profession have very different ways of conceptualizing its nature and sources, determining what is mentally healthy, and deciding what interventions are appropriate. However, regarding the quality of lives, wellbeing is defined as it is used as a positive, ecological concept that comprised of developmental stages throughout the life span, combining physical, psychological, cognitive, social, emotional functions, and also having a subjective sense of satisfaction associated with fulfilling one's potential(2). Well-being includes the competence of comprehending abilities, live a life with purpose and meaning, and make a positive contribution to communities and forming positive relationships with others. It also supports to cope with life's ups and downs and take accountability for oneself as well as for others ${ }^{(3)}$.

*Author for correspondence: <mahjabeenhaque@yahoo.com>. 
For children, the term well-being is explained as the achievement of positive developmental, intellectual, psychological, social, and affective benchmark and by securing attachments, satisfying social relationships and effective adjustment skills(4). So, it is apparent that without some sense of well-being in life, children cannot flourish and reach their full growth. Moreover, parents, teachers, educators, policy makers require the knowledge of child's well-being to help them to achieve their potential and to lead them towards a full-functioning healthy life.

Efforts to promote well-being had been hindered by a lack of valid tools which were suitable for measuring these attributes in the general population. To promote children's well being, the Educational Psychology Service of England collaboration with Stirling Council, has a new scale named Stirling Children's Well-Being Scale (SCWBC) measuring emotional, psychological and social aspects of well-being(5). The SCWBS, positively worded scale, provides a useful instrument for professionals to assess any changes in well-being from a positive psychological perspective. The objective of the scale is to create items which are designed to measure any effects projects and interventions having on well-being, and as such relate to how the participants have been feeling and acting over the last couple of weeks. This scale is intended to be used for screening purposes.

Unfortunately, in Bangladesh there is a scarcity of reliable and valid scales to measure children's well-being. The professionals of our country use some Bangla adapted measures of mental health for their diagnostic practices such as General Health Questionnaire-12(6,7), Psychological Well-being scale(8,9), Beck Youth Inventory ${ }^{(10,11)}$, Warwick-Edinburgh Mental Well-Being Scale(12,13), except Beck Youth Inventory, all are meant for adults. Even Beck Youth Inventory is positive as well as negatively worded items, considering the positive psychology perspective, it is better to focus on positive aspects of well-being rather than a deficit based approach on mental health. As a result, the call for adaptation of the Stirling Children's Well-being Scale (SCWBS) into the cultural context of Bangladesh is felt much needed. The present study aimed to adapt SCWBS into Bangla language and to validate it in our culture through psychometric testing.

\section{Materials and Methods}

A total of 238 participants were chosen covering age range from 10 to 16 years. Among them 115 (48.3\%) were boys and 123 (51.7\%) were girls. The participants were chosen following convenient sampling technique from three different schools.

For the data collection, three materials were used: (i) Demographic and personal information questionnaire, (ii) Original Stirling Children's Well-Being Scale (SCWBS) and (iii) Adapted Bangla version of Beck Youth Inventory of Emotional and Social Impairment Scale. 
The Stirling Children's Well-being Scale (SCWBS) measures the well-being of children between 8 to 16 years age. Items on the scale are rated on a 5-point Likert-based scale ranging from 1 to 5 . The scale is offered for use by educational and health professionals interested in promoting and measuring the emotional development and well-being of children and the effectiveness of interventions.

The Bangla version of Beck Youth Inventory of Emotional and Social Impairment Scale was adapted by Uddin et al. in 2011. The BYI comprises five self-report measures, namely Self-Concept (BSCI), Anxiety (BAI), Depression (BDI), Anger (BANI), and Disruptive Behavior (BDBI) meant for children of age between 11 and 17 years. In this study, first two sub-tests named Self concept (BSCI) and Anxiety (BAI) are used. All items on the scale are rated on a 4-point Likert-based scale ranging from 0 to 3 .

The adaptation of SCWBS involved several multistage procedures following the cross cultural standard guideline regarding adaptation and validation of psychometric instrument of Beaton et al. and Borsa et al. ${ }^{(14,15)}$ as presented in Fig. 1.

Permission was taken from Stirling Council Educational Psychology Service to adapt Stirling Children's Wellbeing Scale (SCWBS). Two independent bilingual translators whose original mother tongue is Bangla translated the original SCWBS.

After that, the researchers compared the two different translations and assessed the semantic, idiomatic, conceptual, linguistic and contextual differences. Then the synthesized version was evaluated by five experts who had the knowledge of what the instrument assessed. These experts evaluated noteworthy features, such as the structure, layout, instrument instructions, and both the scope and adequacy of expressions contained in the items. This assured the linguistic and cultural validity of the adapted scale.

Then blind back translation was done by two experts. The translated versions and the back translated versions were reviewed. The synthesis version was administered to 30 students to check the strength of items concerning their meaning and difficulty. The scale was finalized after this pilot study. The summary of the adaptation process of SCWBS is presented with sample size in Fig. 1.

The final Bangla version of SCWBS was administered to 238 students of three different schools of Dhaka city along with BSCI and BAI. After ten days, re-test was done with 54 students. SPSS ${ }^{\circledR}$ software version 21.0 was used for statistical analysis.

\section{Results and Discussion}

Descriptive statistics of the study variable show that the mean score for all 238 respondents was 57.31 with a standard deviation of 7.587. The lowest possible score is 15 and the highest possible score is 75. This finding is consistent with the original SCWBS's mean (44.14) and SD (7.55) 


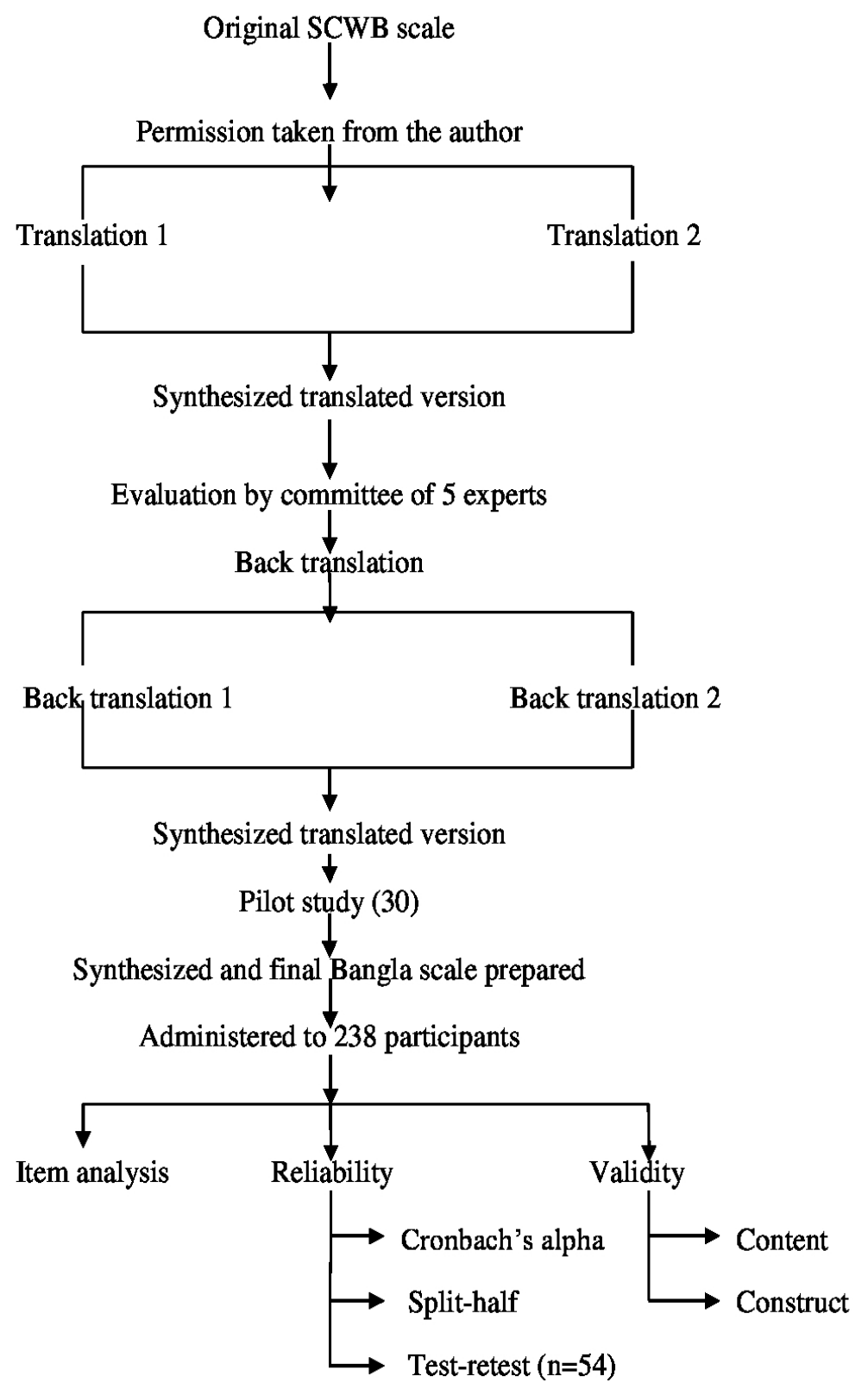

Fig.1. Procedure of Bangla adaptation of SCWBS.

The corrected item-total correlation coefficients presented in Table 1 shows highly significant correlation coefficient values for all items except one item (Item no. 4). This item was revised. High alpha coefficient value (0.746) was indicative of high internal consistency (Table 2). This finding is consistent with the original SCWBS's Cronbach 
alpha value $(0.795)^{(5)}$. The odd-even split-half reliability coefficient value (0.782) states satisfactory level of internal consistency of SCWBS. The test-retest reliability coefficient (0.791) also shows stability of the scale over time.

Table 1. Item-total statistics and Cronbach alpha if the item is deleted.

\begin{tabular}{lcc}
\hline $\begin{array}{l}\text { Items of stirling children's } \\
\text { Well-being Scale }\end{array}$ & $\begin{array}{c}\text { Corrected item-total } \\
\text { correlation }\end{array}$ & $\begin{array}{c}\text { Cronbach's alpha if } \\
\text { item deleted }\end{array}$ \\
\hline SCWBS 1 & $0.430^{*}$ & 0.725 \\
SCWBS 2 & $0.336^{*}$ & 0.735 \\
SCWBS 3 & $0.278^{*}$ & 0.740 \\
SCWBS 4 & 0.161 & 0.752 \\
SCWBS 5 & $0.377^{*}$ & 0.730 \\
SCWBS 6 & $0.317^{*}$ & 0.736 \\
SCWBS 7 & $0.258^{*}$ & 0.741 \\
SCWBS 8 & $0.412^{*}$ & 0.727 \\
SCWBS 9 & $0.364^{*}$ & 0.732 \\
SCWBS 10 & $0.277^{*}$ & 0.742 \\
SCWBS 11 & $0.388^{*}$ & 0.729 \\
SCWBS 12 & $0.379^{*}$ & 0.730 \\
SCWBS 13 & $0.393^{*}$ & 0.728 \\
SCWBS 14 & $0.451^{*}$ & 0.722 \\
SCWBS 15 & $0.421^{*}$ & 0.726 \\
\hline
\end{tabular}

$* p<0.02$.

Table 2. Reliability coefficient of the Bangla version of SCWBS.

\begin{tabular}{llll}
\hline Scale & $\begin{array}{l}\text { Cronbach's alpha, } \\
\text { reliability coefficient } \\
(\mathrm{N}=238)\end{array}$ & $\begin{array}{l}\text { Split-half reliability } \\
\text { coefficient (Spearman } \\
\text {-Brown) }\end{array}$ & $\begin{array}{l}\text { Test-retest reliability } \\
\text { coefficient }(\mathrm{N}=54)\end{array}$ \\
\hline $\begin{array}{l}\text { Stirling children's } \\
\text { well-being scale (SCWBS) }\end{array}$ & $0.746^{*}$ & $0.782^{*}$ & $0.791^{* *}$ \\
\hline
\end{tabular}

** and * Indicate correlation is significant at 0.01 and $0.05 \%$ probability level.

Inter-judge agreement about the content of the Bangla scale was indicative of content validity. Construct validity (Table 3) was tested by correlating the Bangla SCWBS with the two sub-tests of Beck Youth Inventory Scale (Self Concept and Anxiety Inventory) using a Pearson's Bivariate correlation. There was a significant positive correlation between the SCWBS and the BSCI-Y $(r=0.668, \mathrm{~N}=238, \mathrm{p}<0.01)$; a significant negative correlation between the SCWBS and the BAI-Y $(r=-0.350, N=238, p<0.01)$. This suggested a positive correlation between SCWBS and BSCI-Y and lower negative 
correlation between SCWBS and BAI-Y (Table 4). These findings are consistent with the calculated construct validity of original SCWBS, which had a strong positive correlation both with WHO-5(17) $(r=0.742)$ and DuBois Self-Esteem Scale $(r=0.692)^{(5)}$. Both the original and adapted SCWBS showed good external validity.

Table 3. Pearson correlation for construct validity.

\begin{tabular}{lcc}
\hline & $\begin{array}{c}\text { Beck self concept inventory } \\
\text { for youth (BSCI-Y) }\end{array}$ & $\begin{array}{c}\text { Beck anxiety inventory for } \\
\text { youth (BAI-Y) }\end{array}$ \\
\hline $\begin{array}{l}\text { Stirling children's well-being } \\
\text { scale (SCWBS) }\end{array}$ & $0.668^{* *}$ & $-0.350^{* *}$ \\
\hline
\end{tabular}

Fig. 2 shows that scores are normally distributed with a non-significant tail towards the lower end (Skewness $=-0.143, \mathrm{p}>0.05$ ). There was no evidence of floor or ceiling effects in this study.

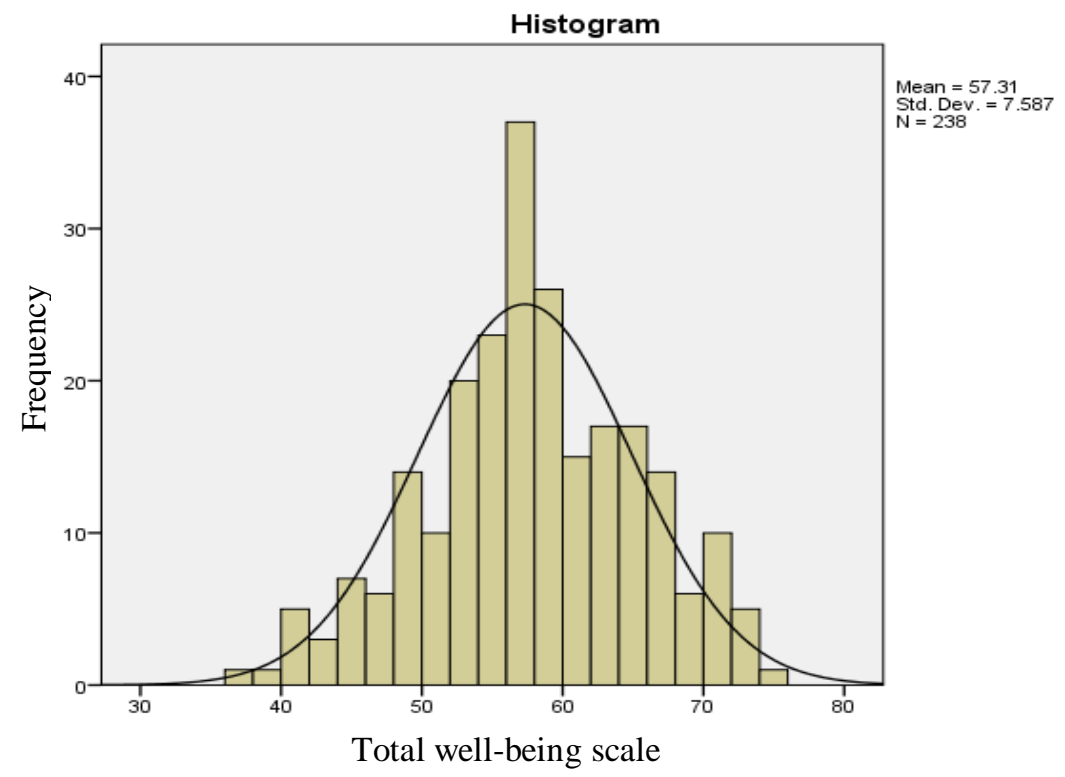

Fig. 2. Score distribution for SCWBS in Bangladeshi samples.

Thus the adapted Bangla scale proved to be a reliable and valid measure of wellbeing meeting the benchmark criteria set out for adapting measures. Overall the scale should provide educational professionals with a concise and robust measure of wellbeing that can assess the effectiveness of projects and interventions for children. The study has a number of recommendations. The instrument needs to be further tested in larger and more diverse populations with qualitative measures. Also with further research the scale's sensitivity and discriminant validity can be established, and from which some diagnostic features may emerge. 


\section{References}

1. Guttman L and S Louis 1982. On the definition and varieties of attitude and wellbeing. Social Indicators Research. 10 (2): 159-174. doi: 10.1007 bf00302508.

2. Pollard EL and L Davidson 2001. Foundations of Child Well-being. Early Childhood and Family Education Unit, UNESCO, Paris.

3. Retrived from http://www.concepts_of_mental_and_social_wellbeing.htm (Retrieved on 16 December, 2015).

4. Office of the Surgeon General 1999. Mental health: A report of the Surgeon General. Rockville, MD: US Department of Health and Human Services.

5. Liddle I and GFA Carter 2015. Emotional and psychological well-being in children: The development and validation of the Stirling Children's Well-being Scale. Educational Psychology in Practice: Theory, research and practice in educational psychology 31: 174185. DOI: $10.1080 / 02667363.2015 .1008409$.

6. Goldberg DP and VF Hillier 1979. A scaled version of the General Health Questionnaire. Psychological Medicine 9: 139-145.

7. Ilyas QSM and S Ayesha 2002. Bangla version of General Health Questionnaires Unpublished Manuscript, Department of Psychology, University of Dhaka.

8. Ryff CD and CLM Keyes 1995. The structure of psychological well-being revisited. Journal of Personality and Social Psychology 69: 719-727.

9. Huque P and HA Begum 2005. Development of scale for measuring psychological well-being for the use in Bangladesh. Bangladesh Psychological Studies 15: 63-74..

10. Beck J, A Beck and Jolly 2001. Beck youth inventories of social and emotional impairment manual. USA: The Psychological Corporation.

11. Uddin MK, AU Huque and AM Shimul 2011. Adaptation of the Beck Youth Inventories of Emotional and Social Impairment for use in Bangladesh. The Dhaka University Journal of psychology 35: 65-80.

12. Tennant R, L Hiller, R Fishwick, S Platt, S Joseph, S Weich and S Stewart-Brown 2007. The Warwick-Edinburgh Mental Well-being Scale (WEMWBS): development and UK validation. Health and Quality of Life Outcomes 5: 63. doi:10.1186/1477-7525-5-63.

13. Rahman ST and MA Imran MA 2013. Bangladeshi Adaptation of Warwick-Edinburgh Mental Well-Being Scale. The Dhaka University Journal of Psychology 37: 49-60.

14. Beaton DE, C Bombardier, F Guillemin and M Bosi-Ferraz 2000. Guidelines for the process of Cross-cultural adaptation of self-report measures. Spine 25: 3186-3191.

15. Borsa JC, BF Damasio and DR Bandeira 2012. Cross-cultural adaptation and validation of psychological instruments: Some considerations. Paidela 22: 423-432.

16. Coolican H 2009). Research Methods and Statistics in Psychology (5th edition). UK: Hodder Education.

17. Bech P 2004. Measuring the dimensions of psychological general well-being by the WHO-5. QoL Newsletter 32:15-16. 